ELECTRONIC LETTER

\title{
No evidence of an association between the T16189C $m+D N A$ variant and late onset dementia
}

\author{
A M Gibson, J A Edwardson, D M Turnbull, I G McKeith, C M Morris, P F Chinnery
}

J Med Genet 2004;41:e7 (http://www.jmedgenet.com/cgi/content/full/41/1/e7)

G enetic factors are important in the aetiology of the two most common neurodegenerative diseases: Alzheimer's disease $(\mathrm{AD})$ and dementia with Lewy bodies (DLB). ${ }^{12}$ Individuals with $\mathrm{AD}$ are more likely to have a similarly affected mother than a similarly affected father, ${ }^{3}$ raising the possibility of a maternally transmitted susceptibility factor. Patients with $\mathrm{AD}$ accumulate cytochrome $c$ oxidase deficient neurones at a faster rate than age matched controls, ${ }^{4}$ and although contentious, abnormal mitochondrial function has been documented in the brains of patients with AD. Cybrid studies suggest that the biochemical abnormality is due to a defect of the mitochondrial genome (mtDNA). ${ }^{5}$ Different mtDNA sequence variants have been associated with $\mathrm{AD}$ and $\mathrm{DLB}$, but there have been no consistent findings, and a maternally transmitted susceptibility factor remains elusive.

The $16.5 \mathrm{~kb}$ mtDNA molecule codes for 13 essential respiratory chain subunits and the 24 RNAs required for intramitochondrial protein synthesis. Transcription and translation of mtDNA is controlled by the short $1 \mathrm{~kb}$ noncoding D-loop. In the wild-type genome, a thymidine residue at nucleotide position (np) 16189 interrupts a tract of cytosine residues between np 16184 and 16193, close to the origin of heavy strand replication $\left(\mathrm{O}_{\mathrm{H}}\right){ }^{6}$ The presence of a cytosine residue at np 16189 generates a homopolymeric C tract which appears to be unstable. It is thought that strand slippage during mtDNA replication generates heteroplasmic length tract variation. Length tract variation at np 16189 has been associated with a variety of different late onset multifactorial disorders, including idiopathic dilated cardiomyopathy, ${ }^{7}$ insulin resistance and type 2 diabetes, ${ }^{89}$ and it has been suggested that the 16189 variant compromises mitochondrial function through an effect on mtDNA replication. ${ }^{9}$ This sequence variant is therefore an attractive candidate for a maternally transmitted susceptibility factor in late onset dementia.

\section{SUBJECTS AND METHODS}

We determined the allele status at np 16189 in DNA extracted from postmortem brains from patients with late onset dementia and age matched controls.

\section{Classification of cases and controls}

Patients were classified as having had AD $(n=182)$ and DLB $(n=97)$ on clinical grounds before death, and the diagnoses were confirmed at autopsy using established neuropathologic criteria. $^{10}$ Control subjects had no clinical evidence of dementia before death and had age associated pathological features with no Lewy bodies $(n=129)$.

\section{Molecular genetic analysis}

The region of the mtDNA D-loop incorporating the np 16189 was amplified by PCR using standard cycling conditions and a fluorescent labelled forward primer (FWD-FAM-tac ttg acc acc tgt agt ac, REV-gga gga tgg tgg tca agg g, Tm $56^{\circ} \mathrm{C}$ ). Half

\section{Key points}

- There is accumulating evidence implicating mitochondrial mechanisms in late onset neurodegenerative disease.

- The T16189C variant of mitochondrial DNA (mtDNA) has been associated with a number of late onset multifactorial disorders, possibly through an effect on mtDNA replication.

- We found no evidence of an association between T16189C and Alzheimer's disease (AD; $n=182$ ) or dementia with Lewy bodies (DLB; $n=97)$ when compared with control subjects $(n=129)$.

of the PCR product was digested overnight with MnlI and run on $4 \%$ 3:1 NuSieve GTG; LE agarose gels, enabling discrimination of the $16189 \mathrm{~T}$ and $\mathrm{C}$ variants based on a characteristic banding pattern. Approximately $10 \%$ of the samples had different banding patterns due to the gain or loss of MnlI sites in the hypervariable regions. These PCR products were sequenced by forward and reverse M13 Big Dye automated sequencing, enabling allele status to be determined at np 16189 in each case. The remaining undigested PCR product was analysed by electrophoresis using an ABI 377 DNA analyser (Applied Biosystems). The size of the PCR product was determined using Genescan software (Applied Biosystems) allowing accurate quantification of the size of the homopolymeric tract associated with np 16189. This was confirmed by forward and reverse sequencing of 20 different DNA samples known to include the range of homopolymeric length tract variants. APOE genotype was determined using a standard procedure.

\section{Statistical analysis}

To minimise the chance of detecting a spurious statistical association we used a logistic regression model to study simultaneously the effect of multiple variables and their interactions when comparing patients with $\mathrm{AD}$ with control subjects and patients with DLB with control subjects. The model assumes that the logarithm of the odds ratio is a linear function of the variables included in the model:

$$
\log \left(\frac{P}{1-P}\right)=B_{0}+B_{1} X_{1}+B_{2} X_{2}+\ldots B n X n
$$

Abbreviations: $A D$, Alzheimer's disease; DLB, dementia with Lewy bodies; mtDNA, mitochondrial DNA; np, nucleotide position 
Table 1 Logistic regression model

\begin{tabular}{|c|c|c|c|c|}
\hline Variable & & $P$ value & Relative risk & $95 \% \mathrm{Cl}$ \\
\hline \multicolumn{5}{|c|}{ AD compared with controls } \\
\hline & Age & 0.053 & 1.03 & $1.00-1.06$ \\
\hline & Sex & 0.092 & 1.60 & $0.93-2.77$ \\
\hline & $A P O E \in 4 /-$ & 0.000 & 5.50 & $2.33-12.99$ \\
\hline & $A P O E \in 3 /-$ & 0.239 & 1.60 & $0.73-3.47$ \\
\hline & $A P O E \in 2 /-$ & 0.026 & 0.43 & $0.21-0.90$ \\
\hline & $16189 \mathrm{C}$ & 0.546 & 0.77 & $0.33-1.79$ \\
\hline & Length heteroplasmy & 0.104 & 2.22 & $0.85-5.81$ \\
\hline \multicolumn{5}{|c|}{ DLB compared with controls* } \\
\hline & Age & 0.128 & 1.03 & $0.99-1.06$ \\
\hline & Sex & 0.144 & 0.64 & $0.35-1.17$ \\
\hline & $A P O E \in 4 /-$ & 0.002 & 4.43 & $1.74-11.31$ \\
\hline & $A P O E \in 3 /-$ & 0.546 & 1.29 & $0.57-2.93$ \\
\hline & $16189 \mathrm{C}$ & 0.997 & 1.00 & $0.40-2.50$ \\
\hline & Length heteroplasmy & 0.867 & 0.92 & $0.34-2.47$ \\
\hline
\end{tabular}

$A D$, Alzheimer's disease; $C l$, confidence interval; DLB, dementia with Lewy bodies.

${ }^{*} A P O E \in 2 /$ - analysis was not carried out because there were no patients with DLB who had an $€ 2$ allele.

Table 2 T16189C allele frequency in the different sub-groups

\begin{tabular}{|c|c|c|c|c|c|}
\hline \multirow{2}{*}{$\begin{array}{l}\text { Status } \\
\text { (mean age, (sd),\% male) }\end{array}$} & & \multicolumn{4}{|c|}{16189 allele status } \\
\hline & & Wild type allele $T$ & Mutant allele C & $\%$ mutant & $95 \% \mathrm{Cl}$ \\
\hline Controls & $\epsilon 4 /-$ & 27 & 7 & 20.6 & $7.0-34.2$ \\
\hline \multirow[t]{2}{*}{$(76.8$ yrs $(9.7), 48 \%)$} & non $\in 4$ & 85 & 8 & 8.6 & $2.9-14.3$ \\
\hline & Total & 112 & 15 & 11.8 & $6.2-17.4$ \\
\hline Alzheimer's Disease & $\in 4 /-$ & 87 & 12 & 12.1 & $5.7-18.6$ \\
\hline \multirow[t]{2}{*}{$(79.0$ yrs $(8.8), 37 \%)$} & non $\in 4$ & 70 & 8 & 10.3 & $3.5-17.0$ \\
\hline & Total & 157 & 20 & 11.3 & $6.6-16.0$ \\
\hline Dementia with Lewy Bodies & $\epsilon 4 /-$ & 47 & 7 & 13.0 & $4.0-22.0$ \\
\hline \multirow[t]{2}{*}{$(78.4$ yrs $(7.3), 55 \%)$} & non $\in 4$ & 37 & 5 & 11.9 & $2.1-21.7$ \\
\hline & Total & 84 & 12 & 12.5 & $5.9-9.1$ \\
\hline
\end{tabular}

Where $P$ is the probability of being affected, $X_{1}, X_{2}, \ldots X_{n}$ represent the chosen predictor variables, and $B_{1}, B_{2}, \ldots B_{n}$ are the coefficients reflecting the nature of each predictor. In both analyses the predictor variables were: age, sex, APOE genotype, 16189 genotype, 16189 homopolymeric tract length heteroplasmy, and homoplasmic homopolymeric tract length variation. This approach also controls for any difference in sex or age distribution between the groups that might confound the analysis or lead to a false positive result. Because of the well established relationship between $A P O E$ genotype and both $\mathrm{AD}$ and $\mathrm{DLB}$, we forced these variables in to the model. All of the remaining variables were then added to the model using stringent forward selection criteria using $\mathrm{F}>4.0$ for inclusion and $\mathrm{F}<4.0$ for rejection, and Minitab v13.1 software (State College, PA). The products were added to the generalised linear model by forward selection if they met the selection criteria.

\section{RESULTS}

Logistic regression analysis confirmed the well established association between $A P O E$ genotype and both AD and DLB, and confirmed that our case and control groups were matched for age and sex. We found no evidence of an association between AD or DLB and the T16189C polymorphism (table 1). Subgroup analysis of the case and control groups by APOE genotype illustrates the even distribution of the T16189C polymorphism between the different groups (table 2 ). We also found no evidence of an association between AD and DLB and homopolymeric tract length heteroplasmy, or homoplasmic homopolymeric tract length variation (table 1).

\section{DISCUSSION}

With the sample sizes used in this study, the power to detect an association conferring an odds ratio of 2.5 at the 0.05 significance level was $>80 \%$ for Alzheimer's disease, and $>70 \%$ for dementia with Lewy bodies. Given the accurate clinical and pathological classification of our study subjects, we therefore conclude that it is unlikely that the T16189C polymorphism or length instability of the 16189 homopolymeric tract is associated with AD or DLB. This cannot, therefore, be an explanation for the increased risk of $\mathrm{AD}$ in patients with a maternal family history of the disorder.

\section{ACKNOWLEDGEMENTS}

The authors are grateful to Dr Andrew Carothers (MRC Human Genetics Unit, Edinburgh) for his statistical advice and the program used to perform the power calculations.

\section{Authors' affiliations}

A M Gibson, J A Edwardson, D M Turnbull, I G McKeith, C M Morris, P F Chinnery, Institute for Ageing and Health, Newcastle General Hospital, Newcastle upon Tyne, UK

D M Turnbull, P F Chinnery, Department of Neurology, The University of Newcastle upon Tyne, UK

This work was funded by the MRC (UK). PFC and DMT receive support from the Wellcome Trust.

Correspondence to: Dr P F Chinnery, Department of Neurology, The Medical School, Framlington Place, Newcastle Upon Tyne, NE2 4HH, UK; P.F.Chinnery@nd.ac.uk

Received 3 June 2003

Accepted 9 July 2003 


\section{REFERENCES}

1 Price DL, Sisodia SS, Borchelt DR. Alzheimer disease-when and why? Nat Genet 1998;19(4):314-6.

2 Ince PG, Perry EK, Morris CM. Dementia with Lewy bodies. A distinct nonAlzheimer dementia syndrome? Brain Pathol 1998;8(2):299-324.

3 Edland SD, Silverman JM, Peskind ER, et al. Increased risk of dementia in mothers of Alzheimer's disease cases: evidence for maternal inheritance. Neurology 1996:47:254-6.

4 Cottrell DA, Blakely EL, Johnson MA, et al. Mitochondrial enzyme-deficient hippocampal neurons and choroidal cells in AD. Neurology 2001;57:260-4. 5 Swerdlow RH, Parks JK, Cassarino DS, et al. Cybrids in Alzheimer's disease: a cellular model of the disease? Neurology 1997;49(4):918-25.
6 Anderson S, Bankier AT, Barrell BG, et al. Sequence and organization of the human mitochondrial genome. Nature 1981;290(5806):457-65.

7 Khogali SS, Mayosi BM, Beattie JM, et al. A common mitochondrial DNA variant associated with susceptibility to dilated cardiomyopathy in two different populations. Lancet 2001:357(9264):1265-7.

8 Casteels K, Ong K, Phillips D, et al. Mitochondrial 16189 variant, thinness at birth, and type-2 diabetes. ALSPAC study team. Avon Longitudinal Study of Pregnancy and Childhood. Lancet 1999;353(9163):1499-500.

9 Poulton J, Luan J, Macaulay V, et al. Type 2 diabetes is associated with a common mitochondrial variant: evidence from a population-based casecontrol study. Hum Mol Genet 2002;11(13):1581-3.

10 Perry R, McKeith I, Perry E. Dementia with Lewy bodies. Cambridge: Cambridge University Press, 1996. 